\section{Growth and Expansion}

$\mathrm{T}$

his is editorial letter number 12, which I submit to you as your Editor-in-Chief of International Surgery.

In my last editorial letter, I indicated that it is always wise to occasionally pause and "take stock" in terms of what we are doing and where we are headed. In so doing, we take the time to gain fresh perspectives and to capitalize on every emerging opportunity in medical and surgical publishing, all with the goal of better serving humanity, via global cooperation and collaboration.

Having taken pause, in this letter, I indicate that we are increasing the frequency of publishing International Surgery. We are receiving new manuscripts at an ever increasing rate, approximately 25 manuscripts per month, requiring more frequent publication. The impetus for manuscript submissions comes from online publishing, the collaboration with PubMed Central and ever improving quality of submitted manuscripts. We also are privileged to have the interest and agreement of additional distinguished reviewers from various countries, who have agreed to serve on our editorial review board. A partial list of the additional institutions and respective countries of new reviewers follows: M.D. Anderson Cancer Center (USA), Sloan-Kettering Cancer Center (USA), Tufts University School of Medicine (USA), Brigham and Women's Hospital (USA), Mayo Clinic (USA), University of Auckland (New Zealand), Prince of Wales Hospital (Hong Kong), Medical Faculty of Martin Luther University (Germany), G.B. Rossi Hospital (Italy), Università degli Studi di Milano
(Italy), Saint Gallen Institute de Oncologia (Brazil), and Lund University Hospital (Sweden).

With our expanding collective group of reviewers, recognition of International Surgery is on an upward trend, with the value and worth of this improving awareness being recognized. As a result, I am delighted to announce that the number of pages in this 98,4 issue of International Surgery is double the number of pages in previous, recent quarterly issues of International Surgery. In the "nutshell", the number of authors who are now requesting rapid reviews by the editorial review board, and, if approved, rapid publishing, is growing.

Given the aforementioned trends, and our solid, fundamental convictions regarding the merits of International Surgery, we will publish on a bimonthly schedule beginning in January, 2014. In so doing, not only will we address emerging publishing trends such as open access publishing, we also will be addressing the desires of those authors who desire more timely publishing of embargoed manuscripts.

As indicated in the past, there is no status quo either a person or entity progresses, or, regresses. As your Editor-in-Chief, I report to you that International Surgery is progressing on a "sound footing" basis.

I thank all of you for your growing support and expanding interest in International Surgery.

Sincerely,

Professor Christopher Chen Editor-in-Chief International Surgery 